\title{
Возрастная динамика структурной организации метакогнитивной сферы личности
}

\author{
Александр А. Карпов \\ Ярославский государственный университет им. П.Г. Демидова, г. Ярославль, Российская \\ Федерация \\ E-mail: karpov.sander2016@yandex.ru
}

\begin{abstract}
Аннотация
Ввеление. Представлены теоретические и эмпирические материалы, раскрывающие специфику возрастной Аинамики структурной организации параметров метакогнитивной сореры личности. Выявлены количественные показатели инАексов структурной организации метакогнитивной сореры мичности А^я различных возрастных групп испытуемых в целях описания общей онтогенетической Аинамики метакогнитивных и метарегулятивных процессов и качеств.
\end{abstract}

Методы. Эмпирическое исследование осуществляется на двух основных и взаимодополняющих Аруг Аруга уровнях. Во-первых, это реализация аналитического уровня исслеАования, направленного на выявление и интерпретацию частных закономерностей Аинамики отАельных ее параметров. Во-вторых, это реализация структурного уровня исслеАования, вкАючающего в себя Ава крупных метолических блока - Аиагностический и математико-статистический, вк^ючая более комплексный - структурно-психологический уровень исслеАования.

Результаты. Впервые выявлены конкретные особенности и закономерности, ^ежащие в основе онтогенетической и генетической Аинамики метакогнитивных и метарегулятивных процессов и качеств ^ичности и зак^ючающиеся в закономерном изменении показателей структурной организации параметров метакогнитивной сореры ^ичности в зависимости от возраста. Основные результаты преАставлены в виде таблицы, структурограмм и графикка.

ОбсужАение результатов. Результаты исследования проанализированы с позиций основных, в том числе - авторских, поАХоАОв К Изучению метакогнитивной сореры ^ичности. Установлено, что на возрастном интервале от 20 Ао $62 \wedge е т$ параметры индивидуальной меры выраженности основных метакогнитивных и метарегулятивных процессов по отлельности не характеризуются значимыми изменениями. В то же время обнаружено, что с возрастом выяв^яется значимое усиление степени структурной организации основных параметров метакогнитивной сореры ^ичности. При увеличении возраста наиболее заметной яв^яется общая Аинамика инлекса когерентности структуры параметров метакогнитивной сореры. Вместе с тем, Аинамика индекса Аивергентности является существенно 


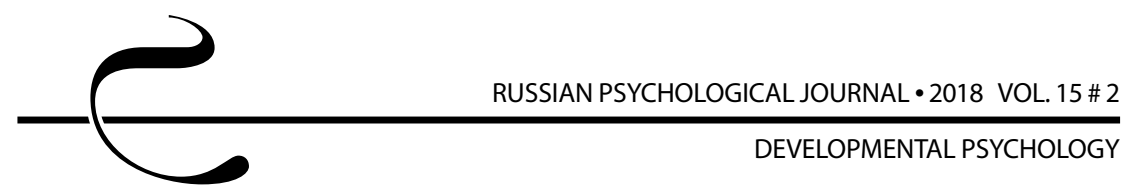

менее выраженной во всех возрастных группах. Наиболее выраженная иинамика характерна Аля показателей индекса общей организованности, в основе которой ^ежат взаимодействие и взаимная соорганизация метакогнитивных и метарегулятивных процессов.

\section{КАючевые слова}

метакогнитивизм, метакогнитивные процессы, метакогнитивные качества, онтогенетическая Аинамика, метарегулятивные процессы, генетическая Аинамика, индексы структурной организации, компенсаторная фуннция, ресурсная фрункция

\section{Основные положения}

в современной метакогнитивной психологии сложилась ситуация, при которой практически отсутствуют исследования, солержательно связывающие основные положения метакогнитивизма и возрастной и генетической психологии, что Аелает объективно необходимым осуществление исследования по выявлению закономерностей возрастной Аинамики метакогнитивной сореры мичности;

- в процессе онтогенетического развития параметры индивидуальной меры выраженности основных метакогнитивных и метарегулятивных процессов не об^аАают значимыми изменениями;

- в основе генетической Аинамики метакогнитивных и метарегулятивных процессов и качеств мичности лежит общее увеличение степени их структурной организации, что, по существу, направлено на реализацию Авух основных когнитивных фрункций - компенсаторной и ресурсной;

- вся совокупность обнаруженных структурных изменений метакогнитивной сфреры личности в процессе онтогенетического развития представляется частным вариантом системогенетического типа развития, т. к. общее увеличение степени ее структурной организации выступает определенным средством ее выражения в качестве системной фрормы как основной Аля психики в целом.

\section{Благодарности}

Исследование выполнено при финансовой поддержке гранта Президента РФ для молодых кандидатов наук, № проекта МК-2796.2017.6, и гранта Российского научного фонда (РНФ), № проекта 16-18-10030.

\section{Для цитирования}

Карпов А.А. Возрастная динамика структурной организации метакогнитивной сферы личности // Российский психологический журнал. 2018. Т. 15, №2. С. 108-132. DOI: 10.21702/rpj.2018.2.5

Материалы статьи получены 23.10.2017 


\title{
Age-Related Dynamics of Structural Organization of the Metacognitive Processes and Traits of Personality
}

\author{
Alexander A. Karpov \\ Demidov Yaroslavl State University, Yaroslavl, Russian Federation \\ E-mail: karpov.sander2016@yandex.ru
}

\begin{abstract}
Introduction. The paper: (a) considers theoretical and empirical studies on the agerelated dynamics of structural organization of the metacognitive processes and traits of personality; (b) focuses on quantitative indices of structural organization of the metacognitive processes and traits of personality for various age groups; and $(c)$ describes general ontogenetic dynamics of metacognitive and metaregulative processes and traits.
\end{abstract}

Methods. An empirical study was carried out at two levels that mutually reinforced each other. Specific patterns of the dynamics of certain parameters of metacognitive processes and traits of personality were identified and interpreted at the analytical level. The structural level of the study included two large methodological modules - namely, the diagnostic and statistical module, and the structural and psychological one.

Results. The study revealed specific characteristics and patterns underlying the genetic and ontogenetic dynamics of metacognitive and meta-regulative personality traits and processes. The indices of structural organization of parameters of the metacognitive processes and traits of personality changed depending on age. Research findings are presented in tables, structuregrams, and graphs.

Discussion. The results of the empirical study were considered in the context of the main approaches to studying the metacognitive processes and traits of personality, including the author's approach. The parameters of the main metacognitive and meta-regulative processes do not change significantly between the age of 20 and 62. However, there is a significant age-related increase in the degree of structural organization of the main parameters of the metacognitive traits and processes of personality. With the increase in participants' age the general dynamics of the index of coherence of the parameters structure of the metacognitive processes and traits is most pronounced. Besides, the dynamics of the divergence index is significantly less pronounced in all the age groups. It is established that the index of overall organization, which is based on the interaction and mutual co-organization of meta-regulative and metacognitive processes, has the most prominent dynamics. 


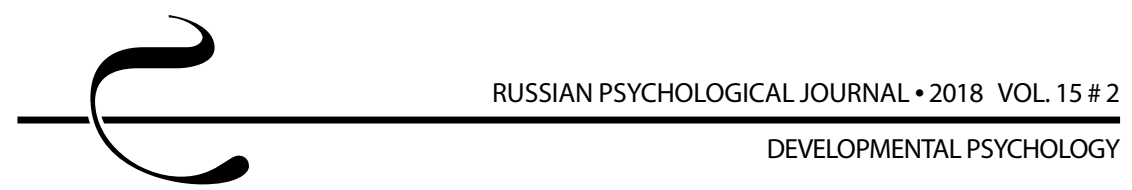

\section{Keywords}

metacognitivism, metacognitive processes, metacognitive traits, ontogenetic dynamics, meta-regulative processes, genetic dynamics, indices of structural organization, compensatory function, resource function

\section{Highlights}

- Given the lack of critical attention to the relationships among basic provisions of metacognitivism, developmental, and genetic psychology, studying the patterns of age-related dynamics of the metacognitive processes and traits of personality is an imperative.

- The individual measures of the extent of manifestation of main metacognitive and meta-regulatory processes do not change substantially in ontogenetic development. The general increase in the degree of the structural organization of metacognitive and meta-regulative processes and traits of personality underlies their genetic dynamics, which essentially enables the implementation of two main cognitive functions - the compensatory and the resource supplying.

- All the discovered structural changes in the metacognitive processes and traits of personality during ontogenetic development represent a specific variation of a systemic genetic development, as the general increase in the degree of its structural organization serves the purpose of its systemic manifestation, which is basic for the psyche as a whole.

\section{Acknowledgments}

The study was supported by the Russian Federation Presidential grant for young candidates of sciences (project no. MK-2796.2017.6) and the Russian Science Foundation grant (project no. 16-18-10030).

\section{For citation}

Karpov A. A. Age-Related Dynamics of Structural Organization of the Metacognitive Processes and Traits of Personality. Rossiiskii psikhologicheskii zhurnal - Russian Psychological Journal, 2018, V. 15, no. 2, pp. 108-132 (in Russian). DOI: 10.21702/rpj.2018.2.5

Original manuscript received 23.10.2017

\section{Введение}

Одной из важнейших задач как общей, так и возрастной психологии выступает установление и интерпретация базовых закономерностей онтогенетической динамики основных психических функций и процессов, прежде всего - когнитивных. Она имеет не только очевидное теоретическое значение, поскольку непосредственно сопряжена с одной из наиболее 
фундаментальных проблем всей психологии - с проблемой генезиса психики, но и столь же большое практическое значение. Последнее связано с тем, что экспликация и объяснение такого рода генетических закономерностей составляют конкретно-научную основу для разработки средств и процедур не только развития и стимулирования когнитивного потенциала личности, но и средств профилактики его регрессивных тенденций, являющихся объективным следствием возрастных изменений. Данная проблема - именно в силу ее объективно высокой значимости - явно «не обделена» вниманием в соответствующих отраслях психологии, а на пути ее решения получен целый ряд важных теоретико-методологических и прикладных результатов. Вместе с тем, традиционно сложилось так, что данная проблема, в основном, соотносится с теми возрастными интервалами, на которых осуществляется преимущественно эволюционное - прогрессивное развитие основных функций и процессов. Другие же, не менее важные фазы онтогенеза - такие, как периоды взрослости и старения, изучены несопоставимо хуже. Кроме того, не все из основных функций и процессов исследованы в равной степени; напротив, имеет место явная диспропорция в сравнительной степени их раскрытия в генетическом плане. Это, в частности, относится и к относительно наиболее сложным из них - к высшим когнитивным функциям и процессам. Несмотря на то, что они являются предметом изучения в достаточно большом количестве исследований, начиная с классических работ школы Б.Г. Ананьева, все же масштаб такого рода исследований не соответствует той роли, которую они играют в общей организации психики и личности [1]. В наиболее общем плане такого рода исследования составляют, как известно, один из основных предметов психологии взрослости, акмеологии и геронтопсихологии, а в более общем плане - важнейшую «составляющую» междисциплинарного научного направления - геронтологии в целом [1, 2, 3, 4, 5].

Наряду с этим, еще одним - также фундаментальным по своей теоретической и практической значимости, но гораздо более «молодым» направлением общей и когнитивной психологии является, как известно, современный метакогнитивизм. Его основным предметом выступает изучение основных метакогнитивных процессов и качеств, а сам он является в настоящее время весьма обширной и очень многоплановой областью исследований $[6,7,8,9$, $10,11,12,13,14,15,16]$. Их своеобразие состоит в том, что они представляют собой такие процессы и качества, которые направлены не на реализацию познавательных, когнитивных функций непосредственно, а на регуляцию и организацию самих этих когнитивных функций. В связи с этим, в качестве ключевого теоретического конструкта в нем выступает понятие «вторичных» процессов (метапроцессов), классическими и наиболее ранними представителями которых являются понятия метамышления и метапамяти $[7,17,18$, 


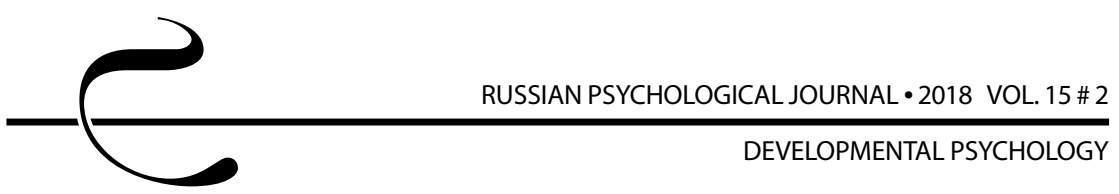

$19,20,21,22,23]$. В собственно методологическом плане метакогнитивизм рассматривается, по мнению многих ведущих исследователей, как один из наиболее крупных «прорывов», имеющий очень существенные теоретические «последствия». Наиболее принципиальным из них является то, что он в очень существенной мере способствует экспликации и объяснению реальной сложности и многоуровневости организации системы психических процессов, иерархичности их организации, а также раскрытию активности субъекта в их регуляции и саморегуляции $[16,20,24,25]$. Они, к тому же, по совершенно понятным причинам являются и наиболее очевидными максимально рельефными и репрезентативными представителями высших психических функций как таковых.

В обоих из отмеченных выше фундаментальных психологических направлениях к настоящему времени получены важнейшие результаты, установлены и проинтерпретированы многие базовые закономерности структурной и функциональной организации психики. Вместе с тем, очень характерной, хотя и не вполне естественной чертой современного состояния исследований в этих направлениях является то, что они оба весьма интенсивно развиваются автономно друг от друга - так сказать, «сами по себе», вне должного содержательного контакта друг с другом. Это положение дел тем более неестественно, и даже не вполне объяснимо, что между ними имеют место многочисленные и очень явные «точки пересечения» их проблематики, взаимосвязь базовых проблем, равно как и фундаментальная общность их основных задач и интерпретационных средств.

Действительно, метакогнитивные процессы, функции и качества как раз и выступают теми высшими проявлениями когнитивной сферы в целом, которые остаются пока недостаточно полно и глубоко раскрытыми в общей и прикладной психологии, в том числе - и в их генетическом плане. Одновременно с этим и сам метакогнитивизм имеет в качестве одной из своих наиболее характерных черт подчеркнутую «автономизированность» своего развития, специфичность своего понятийного аппарата и концептуального строя, явно недостаточное взаимодействие со многими другими важнейшими общепсихологическими направлениями и проблемами. Такая его автономность и «несинтезированность» очень явно прослеживается, прежде всего, именно по отношению к исследованиям собственно генетической направленности, связанным с периодом взрослости и преклонного возраста. Все это является крайне негативным обстоятельством, сдерживающим развитие обоих указанных направлений, а в ряде аспектов - даже практически блокирующим разработку их ключевых проблем. В силу этого, столь же очевидна объективная необходимость в преодолении указанного «разрыва» этих направлений, а в перспективе - и их концептуальный синтез. 
Решение этой - по сути, стратегической и очень общей задачи предполагает необходимость развертывания широкого комплекса исследований, направленных на выявление и интерпретацию конкретных закономерностей генетической динамики основных метакогнитивных процессов и качеств в ходе онтогенеза. Именно они могут составить тот необходимый эмпирический базис, на основе которого такого рода синтез станет реальным. Исходя из этого, основная цель данной работы заключается в том, чтобы попытаться выявить и проинтерпретировать некоторые из такого рода закономерностей, а тем самым содействовать решению сформулированной выше важной как в теоретическом, так и в прикладном отношении общепсихологической проблемы. Наконец, подчеркнем, что наименее раскрытыми являются те особенности генезиса метакогнитивных процессов и качеств, которые сопряжены именно с периодами взрослости и старения; именно это делает их экспликацию особо актуальной.

\section{Методы}

Общая процедура исследования состояла в следующем. Во-первых, при определении «возрастных границ» испытуемых - того онтогенетического интервала, на котором следовало проводить исследования - необходимо учитывать следующее обстоятельство. Совершенно понятно, что существуют два таких онтогенетических интервала, на которых доминирующие тенденции развития метакогнитивной сферы вполне очевидны, хотя и противоположны по своей направленности, равно как и по содержанию происходящих на них генетических трансформаций. С одной стороны, - это, разумеется, ярко выраженная эволюционная фаза, сопряженная, как известно, с периодом развития физиологических и психофизиологических основ психики. С другой стороны, - это столь же явно представленная фаза инволюционного развития, на которой собственно психологические закономерности и механизмы, средства и пр. также приобретают подчиненное значение, а доминирующим «вектором» и объективной детерминантой генетических перестроек вновь становятся факторы собственно физиологического и психофизиологического (даже биологического) плана. Следовательно, необходимо было избрать для исследования тот онтогенетический интервал, на котором динамика параметров метакогнитивной сферы уже выражена, но которая детерминируется, в основном, факторами именно психологического плана. Причем, такой интервал должен быть достаточно большим и охватывать широкий диапазон этапов возрастного развития. В силу этого, он был образован интервалом от 20 до 62 лет. Во-вторых, необходимо было учитывать также, что согласно современным представлениям общее содержание метакогнитивной сферы личности отнюдь не 


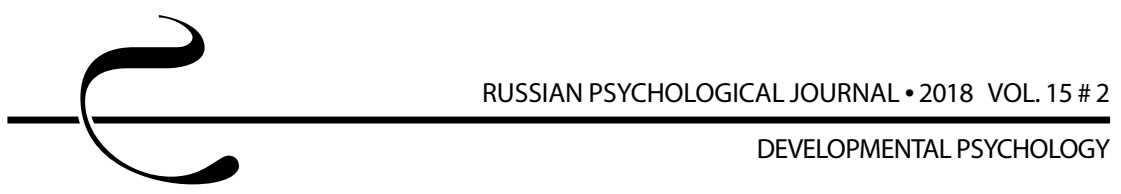

исчерпывается процессами и качествами собственно метакогнитивного плана и не сводится к ним. Наряду с ними, в настоящее время установлены и принципиально иные процессы и образования, также входящие в нее - в частности, не только метакогнитивные, но и метарегулятивные и метакоммуникативные процессы и качества. В силу этого, в качестве предмета исследования следовало взять, по возможности, более широкий состав реально существующих метапроцессов и метакачеств - в том числе и регулятивного, и коммуникативного, и иных планов.

На основе этих двух определяющих в методологическом плане положений и была разработана общая процедура исследования, равно как определены конкретные методы и методики его проведения, а также обработки и интерпретации результатов.

Вначале были сформированы три группы испытуемых, различающихся по параметру возраста - это были группы, состоящие, соответственно, из испытуемых в возрасте 20-22 года, испытуемых в возрасте 40-42 года и испытуемых в возрасте 60-62 года. Численный состав всех групп был одинаковым и равнялся 20-ти испытуемым; выборки были уравнены по половому составу. Естественно, что при формировании такого рода групп с достаточной остротой встает типичная для подобного случая проблема их уравнивания, которая особенно сложна при формировании столь разных в возрастном отношении групп испытуемых. Отчетливо сознавая эту трудность, равно как и практически нереализуемость ее полного устранения, мы, тем не менее, использовали в этих целях наиболее действенный и общепринятый прием. Он состоял в том, что три указанные группы формировались на такой исходной выборке, которая включала в себя лиц, уже обладающих сходством по целому ряду важных социально-демографических и профессиональных характеристик. Конкретно это достигалось тем, что в качестве испытуемых выступали различные возрастные категории лиц, непосредственно причастных к системе вузовского образования; ими были, с одной стороны, студенты и магистранты (первая группа), а с другой - преподаватели двух разных возрастных уровней (вторая и третья группы).

Затем все испытуемые прошли процедуру психодиагностического обследования по специально разработанной «батарее» методик, направленных на определение индивидуальной меры выраженности основных метакогнитивных, метарегулятивных, метакоммуникативных процессов и качеств, а также параметров метапознавательного плана. Диагностировались следующие параметры с применением соответствующих им, наиболее важных и апробированных, ставших общепризнанными в настоящее время, методик:

1) РФи - интегральный (общий) уровень развития рефлексивности (по методике А.В. Карпова, В.В. Пономаревой [8]); 
2) ММ - степень развития метамышления (по методике А.В. Карпова, И.М. Скитяевой [8]);

3) МП - степень развития метапамяти по методике Р. Диксона С. Халтча («Metamemory in Adult» - MIA, по [4]);

4) РФИ - методика Дж. Кагана для диагностики когнитивного стиля «рефлексивность - импульсивность» (по [6]);

5) Маі - метакогнитивная включенность в деятельность (Г. Шроу, Р. Деннисон - по [7]);

6) ММО - метакогнитивный мониторинг (по методике Д. Эверсон, по [8]);

7) СК - методика Г. С. Никифорова, позволяющая диагностировать индивидуальную меру развития процессов самоконтроля;

8) РФс - уровень выраженности социорефлексии (по [7]);

9) Рк - коммуникативная рефлексия (по методике М. Гранта, по [10]);

10) СМП - уровень сформированности «самооценки метакогнитивного поведения» (по методике Д. ЛаКоста, по [11]);

11) 3М - мера сформированности оценки «знаний о мониторинге» (по методике KMAI 3. Тобиаса [16]);

12) 3MC - знание мотивационных стратегий (методика MSLQ, по [6]).

Далее, результаты психодиагностического обследования подвергались обработке в соответствии с двумя основными способами, точнее - на двух основных, разных по глубине и, соответственно, по «разрешающей способности, уровнях обработки. Вначале по отношению к ним применялся известный метод «параллельных профилей». Он представляет собой специальную статистическую процедуру по построению графических изображений исследуемых показателей с целью их сопоставления (как правило, в пределах одной системы координат) и выделения основных отличительных особенностей между ними. Его использование, в свою очередь, равнозначно реализации первого из уровней обработки результатов - аналитического, поскольку на нем возможно установление различий лишь между теми или иными партерами в отдельности. Затем был реализован качественно иной - более «мощный» способ и, соответственно, уровень обработки результатов - cmpуктурно-ncuхологический. Конкретным средством его реализации выступает, как известно, метод вычисления матриц интеркорреляций. Этот метод состоит в том, чтобы определить взаимные корреляции (т. е. интеркорреляции) степени развития исследуемых показателей между собой. В этом случае, как известно, общий массив интеркорреляций представляется в форме матрицы интеркорреляций.

После этого, по отношению к структурограммам, найденным для каждой из трех возрастных групп, был реализован метод определения обобщенных индексов структурной организации (в нашем исследовании - показателей метапроцессов и метакачеств). К ним относятся, как известно, индекс 


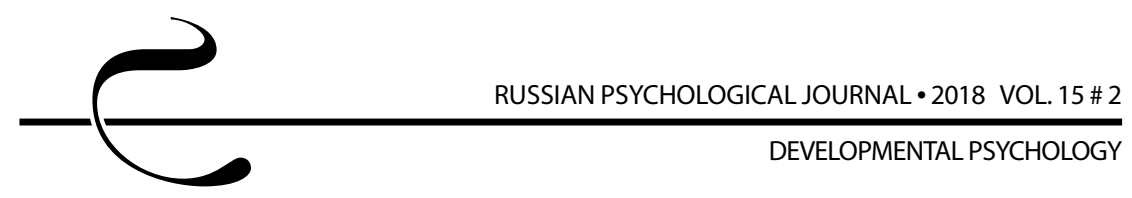

когерентности структуры (ИКС), индекс дивергентности (дифференцированности) структуры (ИДС) и индекс организованности структуры (ИОС). Индекс когерентности структуры параметров определяется как функция числа положительных значимых связей в структуре и степени их значимости; индекс дивергентности структуры (ИДС) - как функция числа и значимости отрицательных связей в структуре; индекс организованности структуры (ИОС) - как функция соотношения общего количества положительных и отрицательных связей, а также их значимости $[6,7,26]$. При этом обычно учитываются связи, значимые при $a=0,01$ (им приписывается «весовой» коэффициент 2 балла) и при $a=0,05$ (им приписывается «весовой» коэффициент 1 балл).

Все полученные по всей структуре «веса» суммируются, что позволяет получить значения указанных индексов. Такой метод позволяет выявить и охарактеризовать детерминацию какого-либо явления не только в плане его «аналитических», «единичных» связей с отдельными индивидуальными качествами, но и в плане его комплексной, структурной обусловленности их целостными подсистемами. В матрицах представлен исчерпывающий комплекс взаимосвязей исследуемых индивидуальных качеств, выраженных в количественных значениях коэффициентов корреляции между ними. Поэтому полученные матрицы позволяют выявить взаимосвязанный комплекс тех или иных качеств и связей между ними (т. е. их структуру) - в нашем случае между показателями метапроцессов и метакачеств.

Наконец, использовался также метод $\mathrm{X}^{2}$ для определения степени гомогенности/гетерогенности матриц интеркорреляций; его содержание, как известно, заключается в следующем: весь массив интеркорреляций, представленных в матрицах, отображается в форме структурограмм (коррелограмм), дающих наглядное представление всех значимо коррелирующих компонентов матрицы. В связи с этим, обычно возникает наиболее частая и типичная задача, заключающаяся в определении того, являются ли какие-либо матрицы (и, соответственно, структурограммы) однородными (гомогенными), или же они разнородны (гетерогенны). На ее решение и направлен данный метод.

\section{Результаты}

Как отмечалось в предыдущем разделе, процедура исследования включала два основных этапа, на первом из которых был реализован аналитический способ - точнее, уровень рассмотрения изучаемой проблемы. В его результате были определены показатели, индицирующие индивидуальную меру развития основных параметров метакогнитивной сферы в отдельности. Затем по отношению к данным, полученным во всех трех возрастных группах, были построены «профили», отображающие всю совокупность найденных значений, и отображены на одной координатной плоскости. Тем самым, по 
отношению к ним был реализован известный метод «параллельных профилей». На основе этих профилей оказывается возможным провести попарное сравнение значений каждого из изучаемых параметров во всех трех группах, а также - в итоге вывить и проинтерпретировать значимые различия между ними. Наиболее общим итогом реализации такого метода (и, соответственно, уровня исследования) были установлены два основных факта.

Во-первых, как это и следовало прогнозировать априорно, с точки зрения наиболее общих и многократно верифицированных в возрастной психологии и физиологии данных, доминирующей, хотя и умеренной тенденцией, явилось последовательное снижение индивидуальной меры выраженности большинства исследованных параметров при переходе от первой группе ко второй и - особенно - от второй к третьей. Вместе с тем, такое снижение заметно менее выражено, нежели можно было бы ожидать: оно не так рельефно, как это описано в литературе по отношению к возрастной динамике «первичных» когнитивных процессов и качеств. Следовательно, уже этот результат вскрывает их определенную специфичность, которая состоит, по-видимому, в том, что регрессивные изменения «вторичных» процессов и качеств в большей мере подвержены каким-либо «демпфирующим» и, скорее всего, компенсаторным воздействиям. Вместе с тем, данный результат пока оставляет открытым вопрос о конкретном содержании этих воздействий, а также лежащих в их основе механизмов (см. далее).

Во-вторых, сравнительный анализ данных в трех возрастных подгруппах выявил также и достаточно незначительное число достоверных в статистическом отношении различий по исследованным параметрам в плане индивидуальной меры их выраженности. Такие различия, хотя, конечно, и имеют место, но носят, как правило, характер тенденции (т. е. значимы лишь при $\mathrm{p}=0,80)$ и не являются ни стабильными, ни систематическими, ни тем более - «повсеместными», т. е. охватывающими собой всю их совокупность.

Наиболее заметны возрастные различия по отношению к параметрам метакогнитивного плана; в относительно меньшей степени - для параметров метарегулятивного плана; наконец, они представлены в относительно минимальной степени по отношению к параметрам метакоммуникативного и метапознавательного плана. Более того, по отношению к двум последним типам параметров по ряду из них имеет место даже возрастание их значений. Тем самым - в известной мере вопреки априорным предположениям - было установлено, что на этом уровне исследования (аналитическом) значения индивидуальной меры выраженности метапараметров не эксплицируют каких-либо систематических и устойчивых различий в их обусловленности фактором возраста. В силу этого возникает следующий вполне закономерный вопрос. Либо этих различий, действительно, нет в сколько-нибудь явном 
виде (что, однако, с очевидностью противоречит подавляющему большинству прочно верифицированных в возрастной психологии и физиологии данных). Либо же эти различия все-таки существуют, причем, в достаточно рельефном виде, но носят существенно более глубинный и имплицитный характер и, соответственно, локализуются на качественно ином детерминационном уровне. В связи с этим, собственно говоря, возникает необходимость перехода именно на такой - более мощный и адекватный природе изучаемого предмета уровень их исследования, т. е. на собственно структурный уровень.

При его реализации были найдены матрицы интеркорреляций исследованных параметров отдельно для каждой из возрастных групп. Затем на их основе были построены структурограммы значимо коррелирующих друг с другом параметров (коррелограммы). Они представлены на рисунках 1-3.

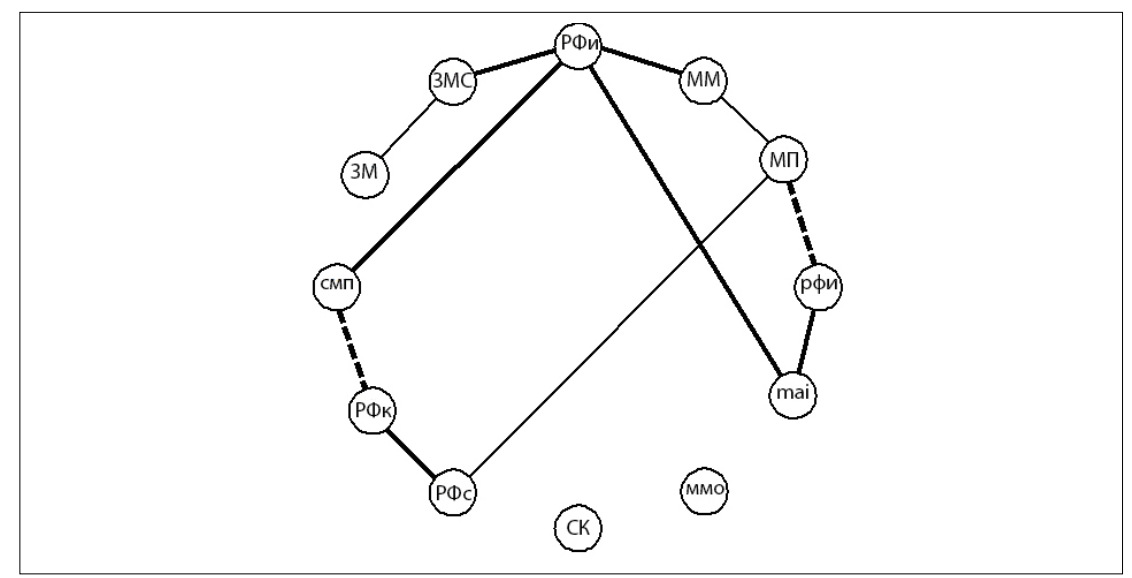

Рисунок 1. Структурограмма метакогнитивных и метарегулятивных процессов и качеств в первой возрастной группе (20-22 гола)

Условные обозначения: РФи - интегральный уровень развития рефлексивности; ММ - показатели развития метамышления; МП - показатели развития метапамяти; РФИ - показатели по методике Дж. Кагана для диагностики когнитивного стиля «рефрлексивность - импульсивность»; Маі - показатели по методике «Метакогнитивная включенность в деятельность»; МMO - показатели по методике диагностики «Метакогнитивный мониторинг»; СК - показатели по методике Г.С. Никифорова, позволяющей диагностировать индивидуальную меру развития прочессов самоконтроля; РФс - уровень выраженности сочиорефллексии; $P_{к}-$ коммуникативная рефлексия; СМП - уровень сформированности «самооценки метакогнитивного поведения»; ЗМ - мера сформированности оценки «знаний о мониторинге»; ЗМС - знание мотивационных стратегий. Жирными линиями обозначены коррелячионные связи, значимые на $p=0,99 ;$ полужирными - связи, значимые на $p=0,95$; пунктирными линиями обозначены отрицательные связи.

Figure 1. Structuregram of metacognitive and meta-regulative processes and traits in the first age group (20-22 years) 


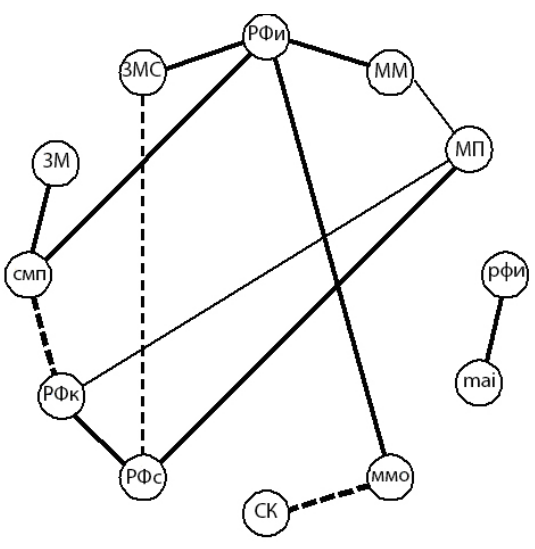

Рисунок 2. Структурограмма метакогнитивных и метарегулятивных процессов и качеств во второй возрастной группе (40-42 гола)

Условные обозначения: те же, что и на рисунке 1.

Figure 2. Structuregram of metacognitive and meta-regulative processes and traits in the second age group (40-42 years)

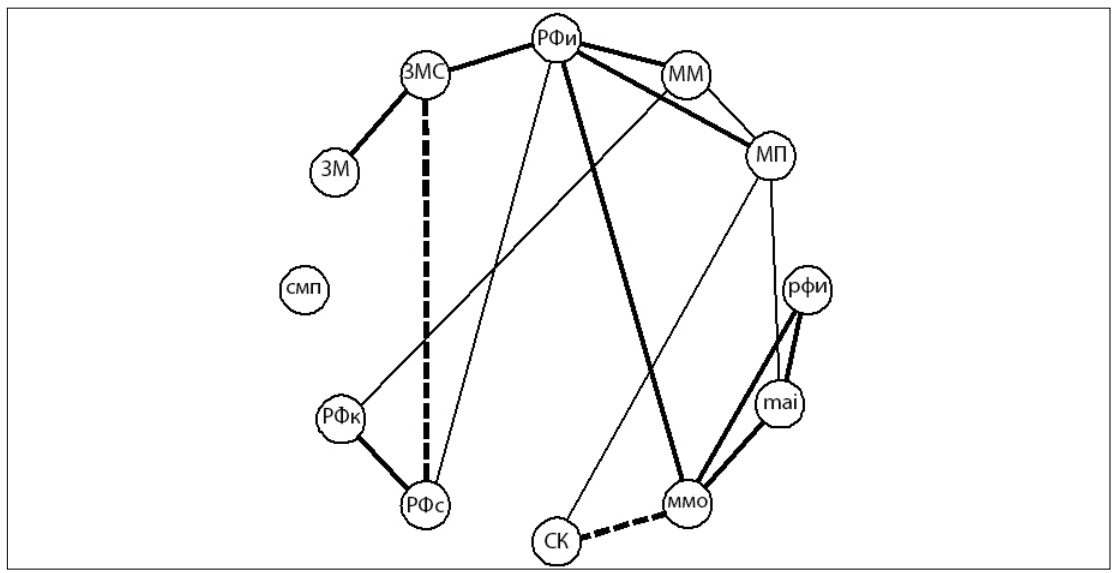

Рисунок 3. Структурограмма метакогнитивных и метарегулятивных процессов и качеств в третьей возрастной группе (60-62 года)

Условные обозначения: те же, что и на рисунках 1, 2.

Figure 3. Structuregram of metacognitive and meta-regulative processes and traits in the third age group (60-62 years) 
Далее, по отношению к ним был реализован метод определения обобщенных структурных индексов, охарактеризованный выше. Он предполагает определение индекса когерентности, показывающего степень интегрированности, синтезированности параметров в целостность; индекса дивергентности, показывающего степень ее дифференцированности, дезинтегрированности; индекса общей организованности, выступающего как суперпозиция двух первых индексов и индицирующего именно общую их организованность, понимаемую как мера преобладания интегративных тенденций над дезинтегративными («дезорганизационными»). Полученные значения индекса представлены в таблице 1, а также на рисунке 4.

Таблица 1. Значения структурных индексов Аля трех возрастных групп

Table 1. Structural indices in three age groups

\begin{tabular}{|l|c|c|c|}
\hline & $\begin{array}{l}\mathbf{2 0 - 2 2} \text { roAa } \\
\mathbf{2 0 - 2 2} \text { years }\end{array}$ & $\begin{array}{l}\mathbf{4 0 - 4 2} \text { roAa } \\
\mathbf{4 0 - 4 2} \text { years }\end{array}$ & $\begin{array}{l}\mathbf{6 0 - 6 2} \text { roAa } \\
\mathbf{6 0 - 6 2} \text { years }\end{array}$ \\
\hline $\begin{array}{l}\text { UKC } \\
\text { ISC }\end{array}$ & 13 & 18 & 23 \\
\hline $\begin{array}{l}\text { UAC } \\
\text { ISD }\end{array}$ & 4 & 5 & 4 \\
\hline $\begin{array}{l}\text { UOC } \\
\text { ISO }\end{array}$ & 9 & 13 & 19 \\
\hline
\end{tabular}

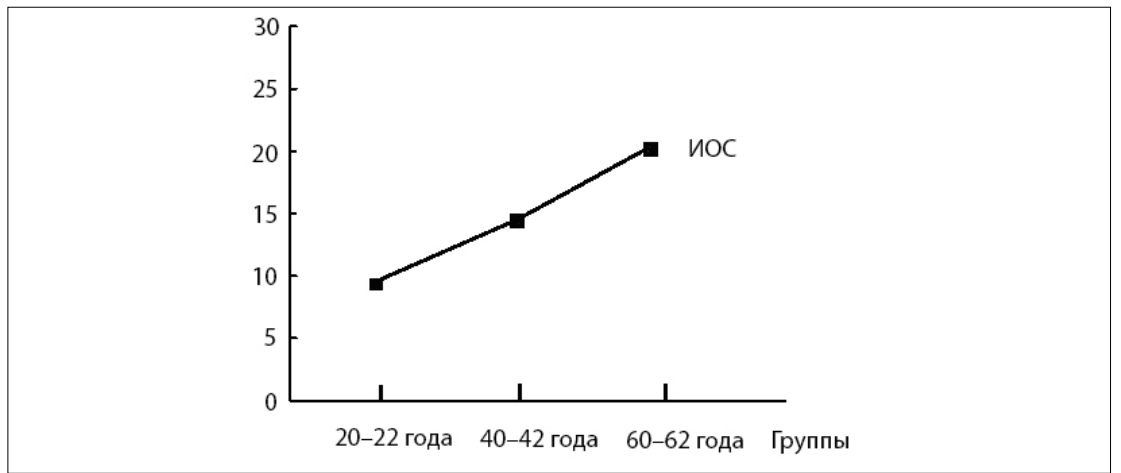

Рисунок 4. Аинамика индекса общей организованности структур (ИОС) метакогнитивных качеств в трех возрастных группах

Примечание: по оси ординат - значения ИОС (в баллах использованной методики).

Figure 4. Dynamics of the index of overall organization of structures (ISO) of metacognitive traits in three age groups

Note: the $y$-axis indicates the ISO scores (in scores of the employed technique). 


\section{Обсуждение результатов}

Анализ всей совокупности представленных выше результатов и, в особенности, данных, представленных в таблице 1, позволяет констатировать следующие основные результаты и эксплицировать те закономерности, которые их обусловливают.

Во-первых, при увеличении возраста испытуемых наиболее выражена динамика индекса когерентности структуры параметров метакогнитивной сферы. Это означает, что доминирующей возрастной тенденцией генетических перестроек данной сферы является все более полная и явная их соорганизация в определенную целостность, их синтезирование во все более когерентную структуру. Можно сказать и более категорично: именно придание совокупности параметров черт структурированности, а значит - и интегрированности, как раз и составляет доминирующий «вектор» этих трансформаций.

Во-вторых, динамика другого индекса - дивергентности - не только заметно менее выражена, но и фактически не представлена в сколько-нибудь явном виде. Кроме того, обращают на себя внимание и весьма малые абсолютные значения этого индекса во всех возрастных группах. На первый взгляд, это не соответствует априорным предположениям, хотя в действительности имеет вполне естественное объяснение, коренящееся именно в специфичности «вторичных» процессов и качеств и их отличиях от «первичных». Суть этой специфики состоит в том, что, как показано в [7], в общей структурной организации именно «вторичных» когнитивных процессов и качеств в очень существенной степени доминируют именно средства и механизмы синтетического, структурообразующего - «соорганизационного» типа. Они в целом, с одной стороны, намного более выражены по сравнению с аналогичными средствами и механизмами дифференцирующего типа; с другой стороны, мера этого преобладания выше, нежели аналогичная разница двух этих тенденций по отношению к «первичным» процессам.

В-третьих, в своем наиболее обобщенном виде доминирование именно этой - интегративной, «синтетической» тенденции (и, соответственно, лежащих в ее основе средств и механизмов) находит свое итоговое и наиболее явное выражение в генетической динамике индекса организованности. Кроме того, поскольку эта динамика, в основном, обусловлена изменениями индекса когерентности, а динамика индекса дивергентности существенно менее представлена, то она в значительной степени подобна ей.

Итак, наиболее общий и принципиальный вывод, который следует из анализа представленных результатов, состоит в том, что с возрастом имеет место значимое и очень явное повышение степени организованности (структурированности, интегрированности) основных параметров метакогнитивной сферы 
личности. При этом различия в значениях наиболее важного структурного индекса - общей организованности третьей и первой групп - измеряются даже отнюдь не «в процентах», а кратно - «в разах»: он выше более чем в 2 раза в третьей группе. Понятно, что такого рода различия не могут быть каким-либо «артефактом», но напротив, свидетельствуют о генетических перестройках достаточно глубинного и принципиального характера. В силу этого становится необходимой попытка их выявления, равно как объяснения ее общего смысла и тех причин, которыми она детерминирована. Прежде чем предпринять ее, отметим, однако, еще один результат, который также будет необходим для нее и который состоит в следующем.

Сравнение найденных матриц и, соответственно, построенных на их основе структурограмм, на предмет их «гомогенности - гетерогенности» по критерию показало их статистически достоверную (при р = 0,95) однородность, т. е. гомогенность. Это означает, что доминирующим «вектором» трансформаций структурной организации исследованных параметров метакогнитивной сферы являются именно количественные изменения ее степени, а не те или иные качественные ее перестройки. Иными словами, общая структурная организация является принципиально инвариантной на всех изученных возрастных интервалах, а изменяется именно степень, мера ее представленности - развитости и совершенства. Сказанное не означает, конечно, что качественные трансформации вообще не имеют место: как раз напротив, они также эксплицируются, но носят своего рода «локальный» характер и состоят, прежде всего, в том, что те или иные структурные связи редуцируются, а на смену им возникают другие. Однако все это происходит при сохранении общего «рисунка» - паттерна их совокупности в целом. Он, хотя и изменяется в своих частных чертах и проявлениях, остается достаточно инвариантным - гомогенным в его общем плане.

Констатируя этот, наиболее общий результат, важно также подчеркнуть, что аналогичные в принципе данные были получены и в еще одном - выполненном нами исследовании [27]. По своему замыслу и процедурной организации оно также было сходным с тем, которое представлено в данной работе. Различия состояли в том, что, во-первых, был исследован иной набор параметров метакогнитивного и метарегулятивного плана; во-вторых, были рассмотрены несколько иные возрастные группы (от 23 до 55 лет). В результате было установлено, что в этом возрастном диапазоне степень структурной организации метакогнитивных и метарегулятивных процессов и качеств, эксплицируемая посредством индекса их общей организованности (ИОС), возрастет в 2,2 раза. Таким образом, можно сделать существенное в плане обоснования корректности и достоверности эксплицированных генетических закономерностей заключение: факт принципиального сходства результатов 
двух независимо проведенных исследований выступает важным средством их взаимоверификации.

Вся совокупность этих результатов допускает, на наш взгляд, следующую - вполне естественную интерпретацию. Как известно, в ходе общего онтогенетического развития дифференцируются три основные фазы: эволюционная (прогрессивная), стабилизационная и инволюционная (регрессивная). Их смысл отражен в названии каждой из фаз, а наиболее исследованной в настоящее время является, разумеется, первая из них, в результате изучения которой установлено множество очень важных закономерностей генетического плана. Вместе с тем, и на двух других фазах также имеют место очень важные и не менее сложные генетические трансформации. Так, показано, что вторая из них - стабилизационная - характеризуется вовсе не тем, что на ней редуцируются собственно трансформационные процессы генетического плана. Напротив, та стабильность (инвариантность) уровневых параметров, которая и проявляется на ней, выступает итоговым эффектом - следствием суперпозиции двух разнонаправленных генетических тенденций. Первая состоит в начинающемся и становящемся все более явным с возрастом снижении потенциала основных психических функций и процессов, равно как и подавляющего большинства иных параметров психики. Вторая тенденция заключается в развитии - причем, также становящемся все более явным с возрастом, определенных средств, направленных на «противодействие» этим негативным изменениям и носящим поэтому, прежде всего, компенсаторный характер. На достаточно длительном интервале онтогенетического развития сравнительная выраженность - так сказать, «сила» этих тенденций - является приблизительно равной, соразмерной друг другу, что и дает в результате относительную сохранность меры выраженности характеристик психических процессов и качеств а, соответственно, и их поддержание на стабильном уровне.

В связи с этой, наиболее общей, закономерностью есть все основания считать, что выявленные в нашем исследовании особенности как раз и являются частным, хотя и достаточно значимым случаем ее действия. Действительно, из общей теории организации, равно как и из целого ряда направлений психологических исследований, известно, что одним из основных средств и даже механизмов повышения и (или) сохранения потенциала той или иной системы, поддержания ее функционального ресурса является именно оптимизация ее структурной организации $[25,28,29,30]$. За счет установления все новых связей между компонентами системы, за счет придания ей все более полной и развитой организованности обеспечивается «прирост» ее возможностей. Кроме того это же выступает и одним из основных средств компенсации снижения функционального потенциала каждого из 


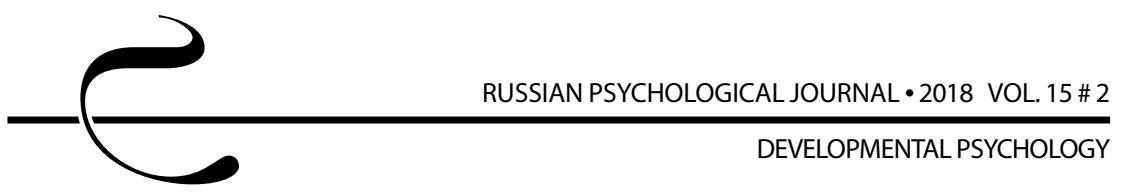

ее компонентов. По-видимому, именно эта, очень общая, закономерность как раз и находит свое проявление в генетических трансформациях метакогнитивной сферы личности. То, вполне естественное снижение потенциала ресурсных возможностей каждого из этих параметров в отдельности, которое происходит с возрастом, компенсируется за счет все более выраженной и, по-видимому, - все более эффективной их структурной организации. При этом между отельными параметрами устанавливаются и упрочиваются связи различного типа - разного конкретного содержания, направленные, однако, на реализацию одной и той же функции - компенсаторной. Эти связи могут носить фасилитирующий характер, когда посредством взаимодействия тех или иных метакогнитивных и метарегулятивных параметров обеспечиваются хорошо известные синергетические эффекты. Вследствие них возникает новое содержание, и порождаются новые возможности, отсутствующие у каждого из взаимодействующих процессов в отдельности. «Классическим» и наиболее известным примером этого является, скажем, мнемотехника как таковая, поскольку ее суть как раз и состоит в том, что средства одних когнитивных процессов (в данном случае мышления и метамышления) реализуются в отношении оптимизации других (памяти и метапамяти), в результате чего существенно повышается общий когнитивный потенциал личности.

Кроме того, эти же связи могут носить и ингибирующий характер: при этом потенциал того или иного метакогнитивного процесса или качества выступает в качестве средства блокировки негативных черт каких-либо других качеств. Так, например, в наших работах $[6,7]$ показано, что одной из важных стратегий метамыслительного плана является выработка и реализация способов предотвращения избыточного рефлексивного контроля над организацией поведения. Это своеобразная блокировка известного феномена «metacognitive loop» (эффекта «метакогнитивной петли»).

Далее, не менее важно и то, что компенсаторная функция не является единственной, поскольку посредством установления развернутой и все более дифференцированной системы структурных связей между метапроцессами и метакачествами у каждого из них также генерируются новые возможности, повышается их собственный потенциал, расширяется их функциональный ресурс. Так, хорошо известно, что сама суть всех метакогнитивных процессов и качеств в том и состоит, что они выступают важными средствами расширения субъектного потенциала - не только когнитивного, но и регулятивного. Например, становление и развитие метамышления (как «вторичного» процесса) позволяет существенно расширить те регулятивные возможности, которыми характеризуется само мышление (как «первичный» когнитивный процесс). Однако у него же появляются новые, дополнительные возможности и в том случае, если оно будет использовать в целях своей собственной 
организации те средства, которые заложены в другом «вторичном» процессе - метапамяти. Конкретным по содержанию, хотя и очень общим по смыслу и функциональной направленности средством этого выступает, как известно, расширение арсенала знаний собственно процедурального типа, являющихся основным содержанием личного опыта в целом и профессионального опыта в частности. В силу этого, можно дифференцировать и еще одну функцию, на реализацию которой направлены все выявленные в нашем исследовании генетические перестройки структурного плана, - ресурсную. Она обеспечивает не только сохранение, но и расширение ресурсного потенциала метакогнитивной сферы личности. Данная функция очень полно, естественным образом, соответствует самой сути и общему функциональному предназначению всех метакогнитивных процессов. Она одновременно может быть проинтерпретирована и как одно из средств оптимизации ментальных ресурсов личности, интенсивно исследующихся в настоящее время.

Далее, следует подчеркнуть, что выявленные структуры метапроцессов и метакачеств оказались статистически достоверно гомогенными по критерию $\chi^{2}$. Это означает, что между ними существуют, прежде всего, именно количественные различия в плане меры, степени их организованности, а не качественные различия. Данный результат, казалось бы, не вполне соответствует тем предположениям, которые можно было бы сформулировать на основе существенности возрастных различий трех групп испытуемых. Более того, по-видимому, было бы гораздо «интереснее и красивее» в теоретическом отношении, если бы эти - качественные различия - все же имели место. Тогда можно было бы, например, сделать «броский» вывод о том, что «с возрастом имеют место качественные перестройки системы метапроцессов и метакачеств». Однако в действительности этого не наблюдается, в чем, тем не менее, заложен важный смысл. Дело в том, что любое развитие, а тем более возрастное, должно характеризоваться его преемственностью и кумулятивным характером тех результатов («достижений»), к которым оно приводит на различных этапах. В особой мере это относится именно к тем базовым, основополагающим средствам и механизмам, на базе которых и осуществляется такое развитие - к психическим процессам и качествам (как «первичным», так и «вторичным»), а также к их общей структуре. Гораздо более эффективным и действенным является именно ее прогрессивная оптимизация и последовательное совершенствование, а не ее «перманентные трансформации». Это и проявилось в полученных нами результатах.

Наконец, следует подчеркнуть, что само становление и прогрессивное развитие каждого из метапроцессов очень органично и также вполне естественным образом сопряжено именно с параметром возраста. Оно выступает 


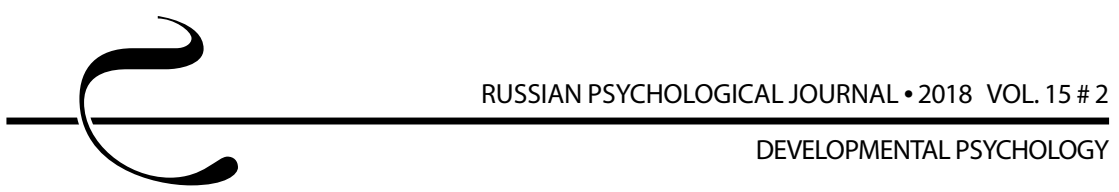

как объективно связанное с этим параметром и, фактически, как производное от него. Дело в том, что те средства и механизмы, которые и лежат в основе каждого из метапроцессов, являются продуктом формирования со стороны самого субъекта - продуктом его личного опыта, результатом, так сказать, «истории его когнитивного развития». Они «приходят с опытом», а потому объективно связаны именно с параметром возраста. Поэтому становление и развитие метапроцессов, с одной стороны, и возрастное развитие, с другой, - это во многом взаимополагаемые и взаимообусловленные явления. В связи с этим становится особенно парадоксальной и не вполне естественной та ситуация, которая сложилась сегодня в метакогнитивизме относительно его связей с генетически-ориентированными исследованиями и которая, как мы отмечали выше, характеризуется очень слабыми связями между ними. И напротив, осознание этого и реализация по отношению к исследованию метакогнитивных и метарегулятивных процессов именно генетического подхода является действенным средством установления не только самих генетических закономерностей, но и новых - дополнительных особенностей, функций и даже механизмов метапроцессов. В свою очередь, это должно рассматриваться как одно из важных проявлений общей гносеологической закономерности: генетически-ориентированные исследования выступают не только в своей прямой - предметной функции, но и в функции метода изучения многих иных предметов психологического исследования.

\section{Выводы:}

1. Одной из наиболее характерных особенностей современного метакогнитивизма, а одновременно и одной из его негативных черт, является его слабая содержательная и концептуальная связь с исследованиями в области возрастной и генетической психологии. Преодоление данной негативной особенности может быть осуществлено на основе раскрытия закономерностей онтогенетической динамики основных метакогнитивных процессов и качеств.

2. В процессе онтогенетического развития на его достаточно длительном интервале (от 20 до 62 лет) параметры индивидуальной меры выраженности основных метакогнитивных и метарегулятивных процессов не характеризуются значимыми изменениями. Хотя и имеет место последовательное снижение этой меры, но оно носит, в основном, характер тенденции. Более того, по отношению к ряду параметров - прежде всего, к метарегулятивным и метапознавательным, имеет место повышение их уровневых характеристик.

3. Основной закономерностью, которая лежит в основе онтогенетической динамики метакогнитивных и метарегулятивных процессов и качеств, является существенное и значимое в статистическом отношении повышение степени структурной организации всей их системы в целом. За счет все 
более полной и развернутой структурной организации обеспечивается не только поддержание общего когнитивного потенциала и функционального ресурса, но и его расширение. Конкретным механизмом этого выступают синергетические эффекты, возникающие вследствие взаимодействия основных метакогнитивных и метарегулятивных процессов.

4. Главным направлением генетической динамики метакогнитивных и метарегулятивных процессов и качеств является повышение степени их структурной организации, а не качественные трансформации их общей структуры. Это является важным условием поддержания стабильности их общей организации, а также объективно необходимым фактором, обеспечивающим преемственность и аккумуляцию метакогнитивного опыта, равно как и средств его получения и закрепления, т. е. самих метакогнитивных процессов.

5. Вся совокупность генетических перестроек системы метакогнитивных и метарегулятивных процессов и качеств в целом, и их структурных трансформаций в особенности, направлена на реализацию двух основных функций - компенсаторной и ресурсной. Первая функция направлена на стабилизацию меры их представленности и минимизацию негативного влияния возрастного фактора. Вторая функция направлена на расширение когнитивного и регулятивного потенциала, являясь тем самым частным, но важным аспектом оптимизации общих ментальных ресурсов личности.

6. Вся совокупность выявленных структурных перестроек метакогнитивной сферы личности, имеющая место в процессе онтогенетического развития, является частным, но важным случаем системогенетического типа развития, поскольку повышение степени ее структурной организованности выступает конкретным средством обретения ею черт собственно системной формы организации как базовой для психики в целом.

\section{Литература}

1. Развитие психофизиологических функций взрослых людей / под ред. Б. Г. Ананьева, Е. И. Степановой. М.: Педагогика, 1977. 200 с.

2. Анцыферова Л. И. Развитие личности и проблемы геронтопсихологии. 2-е изд., испр. и доп. М.: Изд-во Института психологии РАН, 2006. 512 с.

3. Крайг Г. Психология развития / под ред. А. А. Алексеева. СПб.: Питер, 2000. 992 c.

4. Ментальные ресурсы личности: теоретические и прикладные исследования / под ред. М. А. Холодной. М.: Изд-во Института психологии $\mathrm{PAH}, 2016.383 \mathrm{C}$.

5. Психология человека от рождения до смерти / под общ. ред. А. А. Реана. СПб.: ПРАЙМ-ЕВРОЗНАК, 2002. 656 с. 
6. Карпов А. А. Феноменология и диагностика метакогнитивной сферы личности: монография. Ярославль: Изд-во ЯрГУ, 2016. 208 с.

7. Карпов А. А., Карпов А. В. Введение в метакогнитивную психологию. Учебное пособие. М.: Изд-во МПСУ, 2015. 512 с.

8. Карпов А. В., Скитяева И. М. Психология метакогнитивных процессов личности. М.: Изд-во Института психологии РАН, 2005. 352 с.

9. Flavell J. H. Metacognition and cognitive monitoring: a new area of cognitivedevelopmental inquiry // American Psychologist. 1979. Vol. 34, Issue 10. P. 906-911. DOI: $10.1037 / 0003-066 X .34 .10 .906$

10. Metacognition: Cognitive and Social Dimensions / V. Yzerbyt, Lories G. et al. (Eds.) SAGE Publications, 1998. DOI: $10.4135 / 9781446279212$

11. Metacognition: Knowing about Knowing / J. Metcalfe, A. P. Shimamura (Eds.). Cambridge, MA: MIT Press, 1994. 334 p.

12. Metcalfe J., Dunlosky J. Metamemory. New York: Elsevier Ltd., 2008. P. 349-362. URL: http://www.columbia.edu/cu/psychology/metcalfe/PDFs/Metcalfe\%20 2008.pdf (Accessed 08.05.2018).

13. Pressley M., Borkowski J. G., Schneider W. Cognitive Strategies: Good Strategy Users Coordinate Metacognition and Knowledge // Annals of Child Development. Vol. 4. Greenwich, CT: JAI Press, 1987. P. 89-129.

14. Schneider W. The Development of Metacognitive Knowledge in Children and Adolescents: Major Trends and Implications for Education // Mind, Brain, and Education. 2008.Vol.2, Issue 3.P.114-121.DOI:10.1111/j.1751-228X.2008.00041.x

15. Schraw G., Moshman D. Metacognitive theories // Educational Psychology Review. 1995. Vol. 7, Issue 4. P. 351-371.

16. Tobias S., Everson H. T. Knowing What You Know and What You Don't: Further Research on Metacognitive Knowledge Monitoring. Research Report No. 2002-3. N. Y.: College Entrance Examination Board, 2002. 25 p. URL: https://files.eric.ed.gov/fulltext/ED562778.pdf (Accessed 26.05.2018).

17. Cavanaugh J. C., Murphy N. Z. Personality and Metamemory Correlates of Memory Performance in Younger and Older Adults // Educational Gerontology. 1986. Vol. 12, № 4. P. 385-394.

18. Dunlosky J., Serra M. J., \& Baker J. M. C. Metamemory // Handbook of Applied Cognition / F. T. Durso, R. S. Nickerson, S. T. Dumais, S. Lewandowsky, \& T. J. Perfect (Eds.). 2nd ed. UK: John Wiley \& Sons, 2007. P. 137-161.

19. Metcalfe J., Greene M. J. Metacognition of agency // Journal of Experimental Psychology: General. 2007. Vol. 136, Issue 2. P. 184-199. DOI: 10.1037/0096-3445.136.2.184

20. Nelson T. O., Narens L. Metamemory: a theoretical framework and new findings //The Psychology of Learning and Motivation / G. Bower (Ed.). Vol. 26. N. Y.: Academic Press, 1990. P. 125-173. 
21. Implicit Memory and Metacognition / L. M. Reder (Ed.). Mahwah, NJ: Lawrence Erlbaum Associates, 1996. 374 p.

22. Son L. K., Metcalfe J. Metacognitive and control strategies in study-time allocation // Journal of Experimental Psychology: Learning, Memory, and Cognition. 2000, Vol. 26, Issue 1. P. 204-221. DOI: 10.1037/0278-7393.26.1.204

23. Wellman H. M. Metamemory Revisited // Trends in Memory Development Research. Contributions to Human Development. Vol. 9. Basel: Karger, 1983. P. 31-51. DOI: $10.1159 / 000407965$

24. Карпов А. А. Рефлексивность как детерминанта стилей принятия решений в управленческой деятельности // Вестник Ярославского государственного университета им. П. Г. Демидова. Серия Гуманитарные науки. 2017. № 4 (42). С. 82-87.

25. Карпов A. В., Карпова Е. В. Системогенез игровой деятельности: структурно-функциональная организация и генетическая динамика. Т. 3. // Системогенез деятельности. Игра. Учение. Труд: в 4 т. Ярославль: Издво Ярославского государственного университета им. П. Г. Демидова, 2017. 686 c.

26. Карпов А. А. Дифференциальные аспекты структурной организации метакогнитивных качеств личности учащихся высших учебных заведений // Ярославский педагогический вестник. 2017. № 4. С. 218-222.

27. Карпов А. А. Структурно-феноменологический подход в метакогнитивной психологии // Вестник Ярославского государственного университета им. П. Г. Демидова. Серия Гуманитарные науки. 2016. № 4 (38). С. 106-111.

28. Месарович М., Мако Д., Такахара И. Теория иерархических многоуровневых систем. М.: Мир, 1973. 344 с.

29. Системный подход в современной науке / под ред. И. К. Лисеева, В. Н. Садовского. М.: Прогресс-Традиция, 2004. 561 с.

30. Холодная М. А. Психология интеллекта: парадоксы исследования. 2-е изд., перераб. и доп. СПб.: Питер, 2002. 272 с.

\section{References}

1. Anan'ev B. G., Stepanova E. I. (eds.) Razvitie psikhofiziologicheskikh funktsii vzroslykh lyudei [Developing psycho-physiological functions in adults]. Moscow, Pedagogy Publ., 1977. 200 p.

2. Antsyferova L. I. Razvitie lichnosti i problemy gerontopsikhologii [Personality development and the problems of gerontopsychology]. Moscow, Institute of Psychology, RAS Publ., 2006. 512 p.

3. Craig G., Alekseev A. A. (ed.) Psikhologiya razvitiya [Developmental psychology]. St. Petersburg, Piter Publ., 2000. 992 p. 
4. Kholodnaya M. A. (ed.) Mental'nye resursy lichnosti: teoreticheskie i prikladnye issledovaniya [Mental resources of personality: Theoretical and applied research]. Moscow, Institute of Psychology, RAS Publ., 2016. 383 p.

5. Rean A. A. (ed.) Psikhologiya cheloveka ot rozhdeniya do smerti [Human psychology from birth to death]. St. Petersburg, Praim-Evroznak Publ., 2002. 656 p.

6. Karpov A. A. Fenomenologiya i diagnostika metakognitivnoi sfery lichnosti [Phenomenology and diagnostics of the metacognitive processes and traits of personality]. Yaroslavl, Yaroslavl State University Publ., 2016. 208 p.

7. Karpov A. A., Karpov A. V. Vvedenie v metakognitivnuyu psikhologiyu [Introduction to metacognitive psychology]. Moscow, MPSU Publ., 2015. 512 p.

8. Karpov A. V., Skityaeva I. M. Psikhologiya metakognitivnykh protsessov lichnosti [Psychology of metacognitive personality processes]. Moscow, Institute of Psychology, RAS Publ., 2005. 352 p.

9. Flavell J. H. Metacognition and cognitive monitoring: a new area of cognitive-developmental inquiry. American Psychologist, 1979, V. 34, Issue 10, pp. 906-911. DOI: 10.1037/0003-066X.34.10.906

10. Yzerbyt V., Lories G. et al. (eds.) Metacognition: Cognitive and social dimensions. SAGE Publications, 1998. DOI: $10.4135 / 9781446279212$

11. Metcalfe J., Shimamura A. P. (eds.) Metacognition: Knowing about knowing. Cambridge, MA, MIT Press, 1994. 334 p.

12. Metcalfe J., Dunlosky J. Metamemory. New York, Elsevier Ltd., 2008, pp. 349-362. Available at: http://www.columbia.edu/cu/psychology/ metcalfe/PDFs/Metcalfe\%202008.pdf (Accessed 08 May 2018).

13. Pressley M., Borkowski J. G., Schneider W. Cognitive strategies: Good strategy users coordinate metacognition and knowledge. Annals of Child Development, V. 4, Greenwich, CT, JAI Press, 1987, pp. 89-129.

14. Schneider $W$. The development of metacognitive knowledge in children and adolescents: Major trends and implications for education. Mind, Brain, and Education, 2008, V. 2, Issue 3, pp. 114-121. DOI: 10.1111/j.1751-228X.2008.00041.x

15. Schraw G., Moshman D. Metacognitive theories. Educational Psychology Review, 1995, V. 7, Issue 4, pp. 351-371.

16. Tobias S., Everson H. T. Knowing What You Know and What You Don't: Further research on metacognitive knowledge monitoring. Research Report, no. 2002-3. N. Y., College Entrance Examination Board, 2002. 25 p. Available at: https://files.eric.ed.gov/fulltext/ED562778.pdf (Accessed 26 May 2018).

17. Cavanaugh J. C., Murphy N. Z. Personality and metamemory correlates of memory performance in younger and older adults. Educational Gerontology, 1986 , V. 12 , no. 4 , pp. 385-394. 
18. Dunlosky J., Serra M. J., \& Baker J. M. C. Metamemory. In: Durso F. T., Nickerson R. S., Dumais S. T., Lewandowsky S., \& Perfect T. J. (eds.) Handbook of Applied Cognition. UK, John Wiley \& Sons, 2007, pp. 137-161.

19. Metcalfe J., Greene M. J. Metacognition of agency. Journal of Experimental Psychology: General, 2007, V. 136, Issue 2, pp. 184-199. DOI: 10.1037/0096-3445.136.2.184

20. Nelson T. O., Narens L. Metamemory: A theoretical framework and new findings. In: Bower G. (ed.) The psychology of learning and motivation. N. Y., Academic Press, 1990, V. 26, pp. 125-173.

21. Reder L. M. (ed.) Implicit memory and metacognition. Mahwah, NJ, Lawrence Erlbaum Associates, 1996. 374 p.

22. Son L. K., Metcalfe J. Metacognitive and control strategies in study-time allocation. Journal of Experimental Psychology: Learning, Memory, and Cognition, 2000, V. 26, Issue 1, pp. 204-221. DOI: 10.1037/0278-7393.26.1.204

23. Wellman H. M. Metamemory revisited. Trends in memory development research. Contributions to Human Development, V. 9, Basel, Karger, 1983, pp. 31-51. DOI: $10.1159 / 000407965$

24. Karpov A. A. Reflexivity as a determinant of decision-making styles in management. Vestnik Yaroslavskogo gocudarstvennogo universiteta im. P. G. Demidova. Serija Gumanitarnye nauki, 2017, no. 4 (42), pp. 82-87 (in Russian).

25. Karpov A. V., Karpova E. V. Systemic genesis of game activity: Structural and functional organization and genetic dynamics. In: Sistemogenez deyatel'nosti. Igra. Uchenie. Trud: v 4 t. [Systemic genesis of activity: Game, learning, and labour: in 4 volumes]. Yaroslavl, Yaroslavl State University Publ., 2017, V. 3. 686 p.

26. Karpov A. A. Differential aspects of the structural organization of metacognitive personality traits among university students. Yaroslavskii pedagogicheskii vestnik - Yaroslavl Pedagogical Bulletin, 2017, no. 4, pp. 218-222 (in Russian).

27. Karpov A. A. Structural and phenomenological approach in metacognitive psychology. Vestnik Yaroslavskogo gocudarstvennogo universiteta im. P. G. Demidova. Serija Gumanitarnye nauki, 2016, no. 4 (38), pp. 106-111 (in Russian).

28. Mesarovich M., Mako D., Takakhara I. Teoriya ierarkhicheskikh mnogourovnevykh system [The theory of hierarchic multilevel systems]. Moscow, Mir Publ., 1973. 344 p.

29. Liseeva I. K., Sadovskogo V. N. (eds.) Sistemnyi podkhod v sovremennoi nauke [The system approach in modern science]. Moscow, Progress-Traditsiya Publ., 2004. 561 p.

30. Kholodnaya M. A. Psikhologiya intellekta: paradoksy issledovaniya [The psychology of intelligence: Paradoxes of research]. St. Petersburg, Piter Publ., 2002. 272 p. 